\title{
Stokes-Einstein relations and the fluctuation-dissipation theorem in a supercooled colloidal fluid
}

\author{
Daniel Bonn \\ Laboratoire de Physique Statistique, Ecole Normale Supérieure, 24 rue Lhomond, \\ F-75231 Paris Cedex 05, France \\ Willem K. Kegel \\ Van't Hoff Laboratorium, Debye Instituut, Universiteit Utrecht, Padualaan 8, 3584 CH Utrecht, \\ The Netherlands
}

(Received 1 July 2002; accepted 1 November 2002)

\begin{abstract}
The Stokes-Einstein (SE) relation is a form of the fluctuation-dissipation theorem (FDT), and relates the diffusion coefficient of colloidal particles to the viscosity via the thermal energy $k_{B} T$. We study the diffusion coefficient and viscosity of concentrated hard-sphere colloidal model systems in the frequency domain. We show that for these metastable fluid systems strong deviations from the SE relation occur, contrary to predictions of mode coupling theory. Using the FDT, these deviations can be quantified by an effective temperature, $T_{\text {eff }}$. The deviation $T_{\text {eff }} / T-1$ exhibits power-law behavior over several decades in frequency. (C) 2003 American Institute of Physics.
\end{abstract}

[DOI: $10.1063 / 1.1532349]$

\section{INTRODUCTION}

A simple relation between the diffusion coefficient of colloidal particles, $D$, and the solvent viscosity, $\eta_{0}$, is given by the well-known Stokes-Einstein (SE) relation, $D$ $=k_{B} T / 6 \pi \eta_{0} R$. In this equation $k_{B} T$ is the thermal energy and $R$ the radius of a (spherical) colloidal particle. Although strictly valid in diluted systems of colloidal particles only, this relation works remarkably well at higher densities; see for example Ref. 1. Moreover, even molecular systems seem to obey a relation similar to SE; see Ref. 2, and references therein.

There is a close connection between the Stokes-Einstein relation and the more general fluctuation-dissipation theorem (FDT), which states that the response of a system to an external perturbation is the same as that to a spontaneous fluctuation. ${ }^{3}$ The FDT predicts that in equilibrium, the ratio of the correlation and response functions is constant, with the (inverse) temperature as proportionality constant. For the diffusion of colloidal particles, the Einstein relation $D$ $=B k_{B} T$, with $B$ the mobility of the particles, is a manifestation of the FDT and is exact for systems in equilibrium. However, the mobility $B$ of hard-sphere colloids cannot be determined directly, and an additional assumption is necessary to relate the FDT and SE; this is the Stokes relation $B$ $=1 / 6 \pi \eta_{0} R$.

For systems that are not in equilibrium, such as glasses or metastable fluids, there is no a priori reason that the FDT, and therefore also the SE relation, should hold. However, in recent theoretical works ${ }^{4}$ it is proposed to extend the FDT to nonequilibrium (but at most slowly evolving) systems such as glasses. The degree these systems are out of equilibrium can then be characterized by an effective temperature given by the ratio of correlation and response functions. This effective temperature, which has all the properties of a thermodynamic temperature, then reflects the "memory" that glassy systems have from the quench into the glass phase. If this proposal can be justified, it would constitute a first step towards developing a new statistical mechanical description for nonequilibrium systems.

Unfortunately, for most of the systems (structural glasses) studied to date, the correlation and response functions are not easy to obtain experimentally. For this reason, progress has been mainly limited to a number of recent model and simulation (Lennard-Jones glass) studies. ${ }^{5}$ There are, in addition, some experimental indications of a violation of the FDT in glassy systems, but the picture so far is not

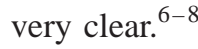

Since the SE relation provides a straightforward relation between the correlation and response functions, which are in addition relatively easy to measure, we study it here for colloidal hard sphere systems close to the glass transition. Colloidal hard-sphere glasses are ideal model glasses, and it is probably fair to say that the strongest evidence for the applicability of the classical mode-coupling theory comes from studies on colloidal glasses. ${ }^{9}$ Recent mode coupling theory calculations have shown that colloidal hard spheres obey a generalized version of the SE relation up to densities very closely approaching the glass transition. ${ }^{10}$ We show here however that for colloidal hard spheres the generalized SE is violated.

Also for molecular systems, there is increasing evidence that close to the glass transition temperature, the SE relation breaks down; see, e.g., Refs. 2 and 11. This breakdown is generally attributed to dynamical heterogeneity when approaching the glass transition; ${ }^{12}$ however direct evidence for this is lacking. The apparent discrepancy with mode coupling theory could be due to the fact that the theory, being effectively a mean-field description of the dynamics of density fluctuations, significantly underestimates dynamical heterogeneities; ${ }^{13}$ see also Refs. 14 and 15 . However, if vio- 
lation of the SE were caused by dynamical heterogeneity, then the timescales should correspond, i.e., breakdown of SE should occur at a similar time scale as the occurrence of dynamical heterogeneity. We show here that for the colloidal hard spheres this is not the case; the SE deviation persists for long times, where heterogeneities become unimportant.

We study the hard-sphere colloidal systems at densities below the glass transition. This is equivalent to thermal systems with temperatures above the glass transition temperature: in athermal systems such as hard spheres, inverse density roughly plays the role of temperature in thermal systems. Hard sphere colloids undergo a true thermodynamic (firstorder) phase transition from a fluid to a (fcc) crystal upon increasing their volume fraction, $\phi .{ }^{16}$ This freezing transition takes place at $\phi=0.494$. In between $\phi=0.494$ and 0.545 , fluid and crystal coexist and at higher densities a singlephase crystal is stable. However, when colloidal hard spheres are rapidly quenched (by centrifugation) from a fluid state to densities above approximately 0.575 , the system is a colloidal glass.

In order to study the relation between diffusion and mobility, we will use here the generalized Stokes-Einstein relation $D(\omega)=k_{B} T / 6 \pi \eta(\omega) R,{ }^{1}$ in which the diffusion coefficient depends on frequency, $\omega$, and the solvent viscosity is replaced by the (frequency-dependent) viscosity of the suspension. This is a natural way to study the relation between diffusion coefficient and viscosity as a function of the time scale.

The "short-time version" of the generalized StokesEinstein relation can then be expressed, in reduced units, as

$$
\eta_{\infty}(\phi)=\frac{1}{D_{s}(\phi)},
$$

where $\eta_{\infty}(\phi)=\eta(\omega \rightarrow \infty)$ is the high frequency limiting shear viscosity in units of $\eta_{0}$, the solvent viscosity. $D_{s}$ is the short-time self-diffusion coefficient in units of $D_{0}$, the diffusion coefficient of the particles at infinite dilution.

Similarly, in reduced units the frequency-dependent Stokes-Einstein relation is, see also Ref. 1,

$$
\eta(\omega)=\frac{1}{D(\omega)} .
$$

In this equation, $D(\omega)=\omega^{2}\left\langle\Delta r^{2}(\omega)\right\rangle / 6 D_{0} \tau_{B}$, where $\omega^{2}\left\langle\Delta r^{2}(\omega)\right\rangle$ is the mean-squared displacement in the frequency domain (i.e., the Laplace-transform of the mean squared displacement in time). $\tau_{B}=R^{2} / 6 D_{0}$ is the Brownian time: the time it takes for a sphere to diffuse over its own radius $R$ in an infinitely diluted system. In this work we test Eqs. (1) and (2) using viscosity and dynamic light scattering data from concentrated suspensions of colloidal hard spheres obtained from different sources in the literature. We do not consider the phase relations between frequency-dependent diffusion and viscosity here, i.e., we will not explicitly evaluate the real and imaginary parts of the viscosity as was done in Ref. 1. However, since the real and imaginary parts of the response are related by the Kramers-Kronig relations, they are not independent functions and knowing the frequencydependence of the viscosity is sufficient. ${ }^{1}$

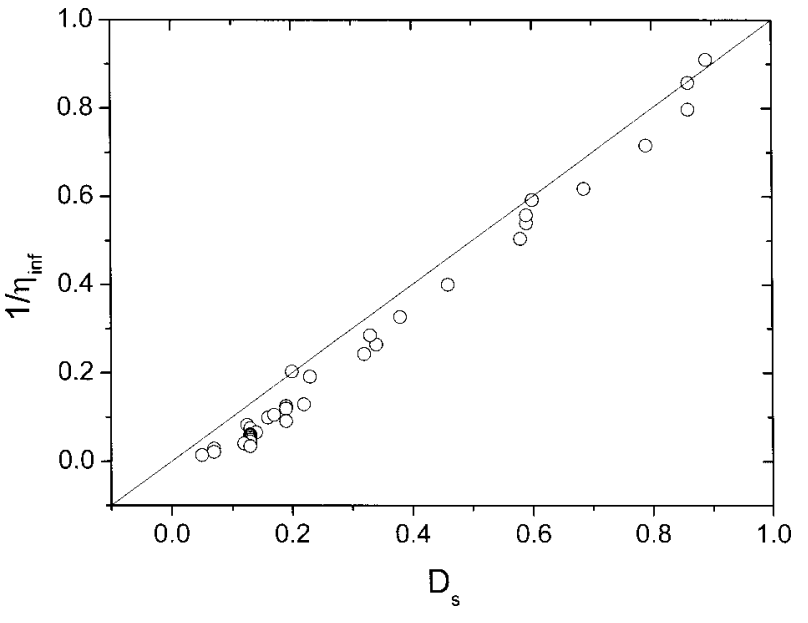

FIG. 1. Short-time data of van der Werff et al. (Ref. 18) (reduced viscosities, i.e., viscosities in units of the solvent viscosity) and van Megen et al. (Ref. 17) (reduced short-time diffusion coefficients).

The key characteristic of the approach of the glass transition is the slowing down of the diffusion of the colloidal particles, which can be characterized by looking at the correlation functions of particle positions by light scattering. The mean squared displacement of the particles as a function of time, $\left\langle\Delta r^{2}(\tau)\right\rangle$, can be extracted directly from the correlation function measured in such experiments. Usually, the results are described in terms of cage-diffusion; the particles are thought to reside in dynamic cages formed by their neighbors. For short times one observes a rapid diffusion within the cage, while for longer times, the particles escape from these dynamic cages. This is the slow process that is responsible for the slowing down of the system near the glass transition; the higher the volume fraction, the more difficult it will be to escape from the cage. This separates the diffusion in a short- and a long-time dynamics, divided (close to the glass transition) by a plateau in the correlation functions. The short- and long-time diffusion coefficient are defined as $D_{S}=\lim _{\tau \rightarrow 0}\left\langle\Delta r^{2}(\tau)\right\rangle / 6 \tau$, and $D_{L}=\lim _{\tau \rightarrow \infty}\left\langle\Delta r^{2}(\tau)\right\rangle / 6 \tau$. In case of dense systems, the short-time diffusion coefficient is sufficiently defined by requiring that time is much smaller than the time where $\left\langle\Delta r^{2}\right\rangle$ reaches a plateau.

\section{RESULTS}

In Fig. 1 we plot the (reduced) short-time diffusion coefficient $^{17}$ as a function of the inverse high shear rate viscosity, ${ }^{18}$ for volume fractions in between 0.032 and 0.59 , covering the whole range from a dilute suspension to a glass. As can be observed in the figure, the data agree with Eq. (1), although the values of the short-time self-diffusion coefficients seem slightly too large, or the viscosities slightly too small. This small but significant deviation at short times has already been reported before and can be attributed to hydrodynamic interactions. ${ }^{19}$ However, in comparison with what follows below for the long-time diffusion, the observed differences are small. We therefore conclude that for short-time diffusion in colloidal hard-sphere systems, the generalized 


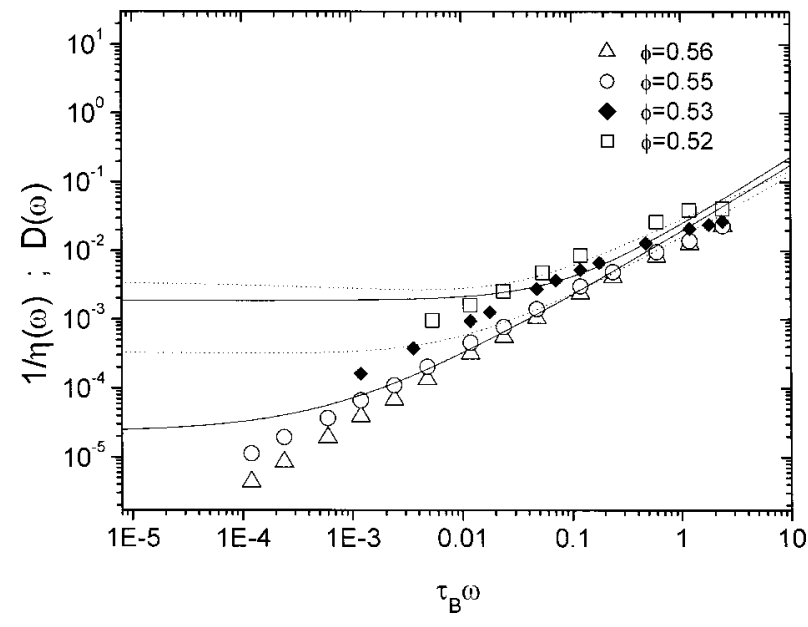

FIG. 2. Viscosity data from Mason and Weitz (Ref. 20) (points) and Laplace-transformed intermediate and long-time diffusion data from van Megen and co-workers (Ref. 17) (lines) as a function of radial frequency $\omega$ made dimensionless by multiplying by the Brownian time $\tau_{B}$. Upper to lower lines in the left hand part of the figure correspond to increasing volume fractions (same as the points). Continuous and broken lines are drawn for the purpose of clarity only.

Stokes-Einstein relation (and thus also the FDT) is reasonably well verified over all experimentally accessible volume fractions.

Clearly, this is not the case for the intermediate- and long-time diffusion, Eq. (2). In Fig. 2, the (inverse of the) frequency dependent viscosity obtained from Ref. 20 is plotted as a function of frequency. The (reduced) viscosity data were calculated from the storage modulus $G^{\prime}(\omega)$ and loss modulus $G^{\prime \prime}(\omega)$ via $\eta(\omega)=\sqrt{G^{\prime 2}(\omega)+G^{\prime \prime 2}(\omega)} / \omega$. These data correspond to the intermediate to long-time diffusion regime. ${ }^{21}$ The prediction of Eq. (2) is then that the inverse reduced viscosity and reduced particle displacements are one and the same thing. We therefore plot in the same figure the reduced particle displacement in frequency space, obtained from the Laplace transform of the mean-squared displacement from Ref. 17. For convenience, the latter were fitted to a function of the form $\left\langle\Delta r^{2}(\tau)\right\rangle=a+b_{1} \tau^{1 / 3}+b_{2} \tau^{2 / 3}+b_{3} \tau$ (where $\tau$ is in units of the Brownian time) in the intermediate- to long-time regime and subsequently Laplace transformed to the frequency domain. The functional form chosen agrees quantitatively with the data over several decades in time, and the last term assures long-time limiting behavior. An independent check of this is that the coefficients $b_{3}$ indeed were always close (to within a few percent) to the long-time diffusion coefficients. ${ }^{17}$

It is clear from the figure that the generalized SE equation [Eq. (2)] gives a very satisfactory description of the relation between diffusion and viscosity at the largest values of $\tau_{B} \omega$. These frequencies are still much smaller than those of Fig. 1; they correspond to the intermediate regime of $\left\langle\Delta r^{2}(\tau)\right\rangle$. Just before the onset of long-time diffusion behavior (roughly at the end of the plateau in $\left\langle\Delta r^{2}(\tau)\right\rangle$ ), the viscosity and diffusion data start deviating. For the long-time regime, significant deviations from Eq. (2) are observed; the values of the inverse viscosity are significantly smaller than the values of the diffusion coefficients.

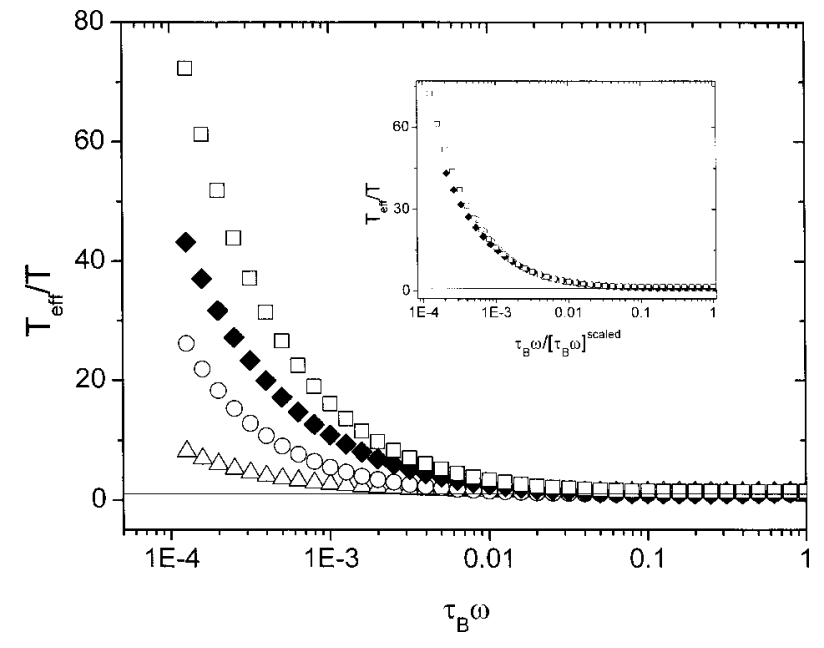

FIG. 3. Effective temperature [as defined by Eq. (3)] as a function of frequency. Inset: Same as the main figure, but the frequencies have been divided by $\left[\tau_{B} \omega\right]^{\text {scaled }}=1(\phi=0.52), 0.6(\phi=0.53), 0.23(\phi=0.55)$, and $0.048(\phi=0.56)$.

As will be argued in the Discussion, there is no reason to believe that the Stokes relation, which relates the mobility of the particles to the viscosity, breaks down. We therefore quantify the deviation from the generalized SE relation [a deviation from the FDT (Ref. 5)] by defining an effective temperature as

$$
\frac{T_{\mathrm{eff}}(\omega)}{T}=\eta(\omega) D(\omega) .
$$

From Eq. (2), the product $\eta(\omega) D(\omega)$ equals unity if the SE is obeyed; in this case the effective temperature equals the real temperature. In order to deduce an effective temperature, equally spaced data are required. To this aim, the viscosity data of Fig. 2 were fitted to functions of the form $\log (\eta)$ $=A+B_{1} \log \left(\tau_{B} \omega\right)+B_{2}\left[\log \left(\tau_{B} \omega\right)\right]^{2}$. The coefficients $B_{1}$ determine the values of $\eta$ in Fig. 3. Fitting the data, we find that to a good approximation $B_{1}(\phi) \approx-13 \phi+6.39$, so that $B_{1}$ $=0$ when $\phi \approx 0.491$, which is very close to the freezing volume fraction of $\phi=0.494$. Since $D(\omega)$ in Eq. (3) becomes constant in the long-time diffusion limit, this immediately implies that for volume fractions smaller than the freezing density, the effective temperature no longer depends on frequency.

Figure 3 depicts the results; for high frequency, $T_{\text {eff }} / T$ $\approx 1$ is found, as would be expected. On the other hand, for small frequencies, the "FDT temperature" depends on frequency, and an important difference with the ambient temperature develops. This behavior can also be inferred from Fig. 2: the diffusion data reach a plateau while viscosity keeps increasing upon decreasing frequency.

Interestingly, the data of Fig. 3 collapse onto a single curve by rescaling the frequencies, leading to a "master curve" that is independent of the volume fraction. This "rescaling" was carried out as follows: If the data for $\phi=0.52$ are taken as a reference, then the data for $\phi=0.53,0.55$, and 0.56 fall onto the reference curve if their $\tau_{B} \omega$ values are divided by $\left[\tau_{B} \omega\right]^{\text {scaled }}=0.6,0.23$, and 0.048 , respectively. This is shown in the inset of Fig. 3. From the data, it follows 


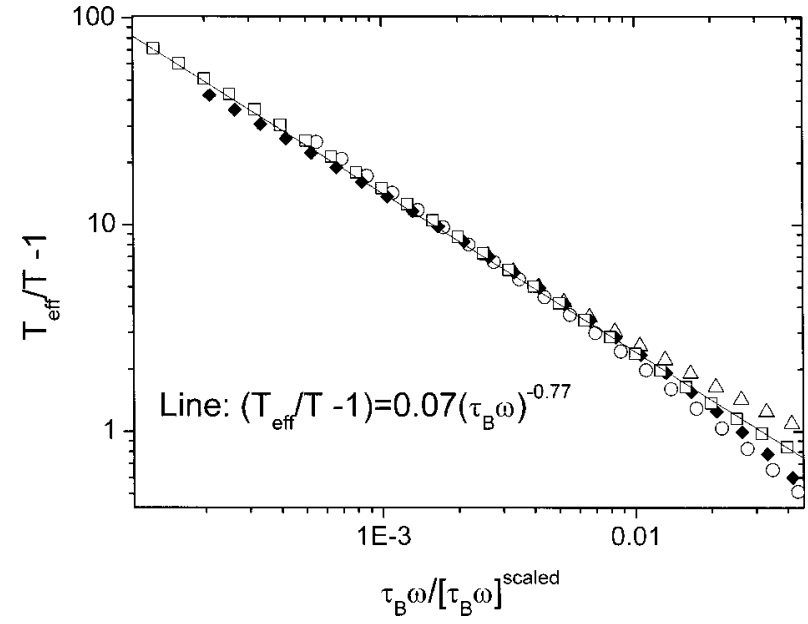

FIG. 4. $\left(T_{\text {eff }} / T\right)-1$ vs rescaled frequencies (as in Fig. 3, inset) in the loglog plane.

that $\ln \left(\left[\tau_{B} \omega\right]^{\text {scaled }}\right)=(37 \pm 7)-(40 \pm 8)\left(\phi / \phi_{g}\right)$ (with $\phi_{g}$ is the volume fraction that corresponds to the glass transition, taken as 0.575). This behavior is equivalent to the Arrhenius behavior observed for (certain) molecular glass formers. Thus, although our experimental window is rather limited, it appears that the observed rescaling with the volume fraction derives directly from the approach of the glass transition.

In Fig. 4 we plotted the deviation, $\left(T_{\text {eff }} / T\right)-1$, as a function of the rescaled frequencies. The interesting observation is that the data follow a power law with $T_{\text {eff }} / T-1 \propto\left(\tau_{B} \omega\right)^{\alpha}$, with $\alpha=-0.77 \pm 0.05$. As far as we know, no predictions are available so far for this power law, although a continuous variation of the temperature with frequency such as we find here is consistent with certain model calculations and some of the experiments. ${ }^{8,11}$ More importantly, this power-law behavior shows that characterizing the nonequilibrium system with a single effective temperature that follows from the FDT is not sufficient; an infinite number of effective temperatures is found.

\section{DISCUSSION AND CONCLUSION}

We find that the generalized SE equation [Eq. 2] gives a very satisfactory description of the relation between diffusion and viscosity at relatively high frequencies, i.e., down to values of $\tau_{B} \omega$ corresponding to the intermediate regime of $\left\langle\Delta r^{2}(\tau)\right\rangle$ (Figs. 2 and 3). Just before the onset of long-time diffusion behavior (roughly at the end of the plateau in $\left.\left\langle\Delta r^{2}(\tau)\right\rangle\right)$, the viscosity and diffusion data start deviating. For the long-time regime, significant deviations from the SE relation are observed, suggesting that as soon as a slow mode develops upon approach of the glass transition, the SE relation is violated. At these relatively small frequencies, the values of the inverse viscosity are significantly smaller than the values of the diffusion coefficients. This implies that, upon the approach of the glass transition, momentum (i.e., viscosity) is more efficiently transported than mass on long time scales. In the "cage" picture of glassy dynamics an intuitive explanation would be that the particle can transmit momentum by collisions with its neighbors, but remains trapped in an effective cage consisting of those same neighbors. However, this (simple) picture does not apply for the longest time scales in systems with the lowest volume fractions, where inverse viscosity and diffusion coefficients keep deviating. In that case the particles are expected to have escaped these cages. A possible explanation is ${ }^{14}$ that even for long-time diffusion, the distinct Van Hove correlation function still shows significant memory of local structure.

Mode-coupling theory ${ }^{10}$ predicts that for both intermediate- and long-time diffusion, the generalized SE relation provides a good approximation for hard-sphere systems over the range of frequencies considered here; the difference between the mode coupling theory and the generalized Stokes-Einstein relation is at most 20\%. This also implies that the Stokes relation should remain valid for this system. If the mode-coupling theory fails, this is likely to be due to dynamical heterogeneity, as mentioned in the Introduction section. Measurements show that dynamical heterogeneity is most prominent in these systems ${ }^{14}$ close to the long time diffusion regime, just where the SE relation breaks down. However, dynamical heterogeneity disappears over longer time scales ${ }^{14}$ whereas the deviation from the SE relation becomes more pronounced. Therefore our results suggest in addition a violation of the more general FDT.

A possible caveat in our results might be that the different behavior is caused by subtle differences in the model systems as used in Refs. 17 and 20. In the system studied by Mason and Weitz, ${ }^{20}$ size polydispersity is approximately $20 \%$ while in the other system ${ }^{17}$ it is about $5 \%$. However, it is expected that larger polydispersity leads to an increasing mobility and thus an increasing inverse viscosity. In fact, the opposite effect is observed in Fig. 2. Another subtle effect may be that in concentrated hard-sphere suspensions, very small differences in volume fraction result in significantly different relaxation times. Although this might in principle explain the deviations for the largest volume fractions of 0.55 and 0.56 , it does not account for the very similar behavior observed for the smallest volume fractions. For these solutions, the relaxation times much less sensitively depend on volume fraction. This implies a nontrivial reason for the observed behavior, which we interpret as a breakdown of the $\mathrm{SE}$ relation.

In conclusion, we have shown that for a supercooled colloidal hard-sphere fluid even before the glass transition, the SE relation is violated. This may in fact not be so surprising, as the glass transition in hard-sphere systems, and possibly all systems, is not a well-defined (thermodynamic) transition. $^{22}$ The more surprising observation is that the results do not agree with detailed mode-coupling calculations of Banchio et al. ${ }^{10}$ for exactly the same system. One reason for this discrepancy might be due to the fact that experimental systems are more dynamically heterogeneous than predicted by mode coupling theory. The time scales at which the SE breaks down correspond to the time scales where dynamic heterogeneity is most prominent. Yet the systems keep deviating from the SE relation, even after longer times, where dynamic heterogeneity decreases.

Therefore our results suggest a violation of the more 
general FDT. If this were true, it may be related to the fact that the systems are metastable since all densities are above the freezing density. This, in turn, suggests that violation of the FDT is a sensitive indicator of metastability and our result may not only be relevant from a fundamental point of view, but may also have impact in applications; so far, the only way of experimentally characterizing metastability is to simply wait and see if a system changes.

\section{ACKNOWLEDGMENTS}

The authors are grateful to Bill van Megen for kindly providing his data, and to Leticia Cugliandolo, Gerhard Nägele, Gijsje Koenderink, Jacques Meunier, Jorge Kurchan, and Jean-Louis Barrat for discussions and comments.

${ }^{1}$ T. G. Mason and D. A. Weitz, Phys. Rev. Lett. 74, 1250 (1995); G. H. Koenderink and A. P. Philipse, Langmuir 16, 5631 (2000).

${ }^{2}$ C. DeMichelle and D. Leporini, Phys. Rev. E 63, 036701 (2001).

${ }^{3}$ S. R. de Groot and P. Mazur, Non-Equilibrium Thermodynamics (Dover, New York, 1984); R. Kubo, M. Toda, and N. Hashitsume, Statistical Physics II (Springer, Berlin, 1995).

${ }^{4}$ L. F. Cugliandolo, J. Kurchan, and L. Peliti, Phys. Rev. E 55, 3898 (1997);

L. F. Cugliandolo and J. Kurchan, Prog. Theor. Phys. 126, 407 (1997).

${ }^{5}$ W. Kob and J.-L. Barrat, Phys. Rev. Lett. 78, 4581 (1997); G. Parisi, ibid. 79, 3660 (1997).

${ }^{6}$ T. Grigera and N. Israeloff, Phys. Rev. Lett. 83, 5038 (1999).

${ }^{7}$ L. Bellon, S. Ciliberto, and C. Laroche, Europhys. Lett. 53, 511 (2001); L. Bellon and S. Ciliberto, Physica D 168, 325 (2002).

${ }^{8}$ D. Hérisson and M. Ocio, Phys. Rev. Lett. 88, 257202 (2002).
${ }^{9}$ P. N. Pusey and W. van Megen, Phys. Rev. Lett. 59, 2083 (1987); W. van Megen, S. M. Underwood, and P. N. Pusey, ibid. 67, 1586 (1991); W. van Megen and S. Underwood, ibid. 70, 2766 (1993); W. Poon, P. Pusey, and H. Lekkerkerker, Phys. World 9, 27 (1996).

${ }^{10}$ A. J. Banchio, G. Nägele, and J. Bergenholtz, J. Chem. Phys. 111, 8721 (1999).

${ }^{11}$ C. Z. W. Liu and I. Oppenheim, Phys. Rev. E 53, 799 (1996).

${ }^{12}$ H. Sillescu, J. Non-Cryst. Solids 243, 81 (1999); M. T. Cicerone, F. R. Blackburn, and M. D. Ediger, J. Chem. Phys. 102, 471 (1995).

${ }^{13}$ M. Fuchs, W. Götze, and M. R. Mayr, Phys. Rev. E 58, 3384 (1998).

${ }^{14}$ A. Kasper, E. Bartch, and H. Sillescu, Langmuir 14, 5004 (1998); T. C. Mortensen and W. van Megen, in Slow Dynamics in Complex Systems, AIP Conference Proceedings (Fukuoka, Japan, 1998); W. K. Kegel and A. van Blaaderen, Science 287, 290 (2000); E. R. Weeks, J. C. Crocker, A. C. Levitt, A. Schofield, and D. A. Weitz, ibid. 287, 672 (2000).

${ }^{15}$ The prediction, by MCT (Ref. 13), of the so-called non-Gaussian parameter, $\alpha_{2}$, being a measure for dynamical heterogeneity, is roughly an order of magnitude too small when compared to experiments (Ref. 14).

${ }^{16}$ W. W. Wood and J. D. Jacobsen, J. Chem. Phys. 27, 1207 (1957); B. J. Alder and T. E. Wainwright, ibid. 27, 1208 (1957).

${ }^{17}$ W. van Megen, T. C. Mortensen, S. R. Williams, and J. Müller, Phys. Rev. E 58, 6073 (1998).

${ }^{18}$ J. C. van der Werff, C. G. deKruif, C. Blom, and J. Mellema, Phys. Rev. A 39, 795 (1989).

${ }^{19}$ J. Bergenholtz, F. M. Horn, W. Richtering, N. Willenbacher, and N. J. Wagner, Phys. Rev. E 58, R4088 (1998); J. C. Ladd, J. Chem. Phys. 93, 3484 (1990).

${ }^{20}$ T. G. Mason and D. A. Weitz, Phys. Rev. Lett. 75, 2770 (1995).

${ }^{21}$ With "intermediate" we refer to the plateau of the correlation functions that is observed in concentrated (for hard spheres $\phi>0.51$ ) colloidal suspensions.

${ }^{22}$ L. Santen and W. Krauth, Nature (London) 405, 550 (2000). 